\title{
Friction without contact
}

Perhaps few physical concepts have received less attention relative to their importance than friction. Learning the 'effective' notions of static and sliding friction, students of mechanics can easily mistake our ignorance of the phenomenon for deep knowledge. What we do know is that macroscopic friction often depends sensitively on microscopic molecular details. We might naively expect rough surfaces to show greater friction, for example, yet it is sometimes higher between perfectly smooth and molecularly clean surfaces.

More surprising, friction can exist even between bodies that make no contact whatsoever. For example, measurements of the force required to drag the aluminium tip of a scanning tunnelling microscope over a gold surface estimate a friction force, linear in velocity, of the order of $10^{-11} \mathrm{~kg} \mathrm{~s}^{-1}$. Many other experiments, including recent work with polymer thin films, generally confirm the existence of a non-contact friction force between surfaces separated by very small distances.

What's behind it? As with many mysteries of physics, this one seems to have quantum origins, with close links with other seemingly 'anomalous' behaviour of bodies when held close together.

By the Stefan-Boltzmann law, we'd normally expect the heat flow between two blackbodies at temperatures $T_{1}$ and $T_{2}$ to be proportional to $T_{2}^{4}-T_{1}^{4}$. For two infinite planar bodies, the heat transferred does not depend on the distance between the bodies. But this result depends on the properties of radiation in the farfield, and experiments demonstrate that the heat flow is indeed different if the distance between the bodies is less than about $\lambda_{\mathrm{T}}=c \hbar / \mathrm{kT}$, the typical wavelength of the thermal radiation, when near-field effects and photon tunnelling influence the flow dramatically. Experiments over the past decade have shown that photon tunnelling can enhance heat exchange between conductors by several orders of magnitude even at room temperature.

What does this have to do with 'non-contact' friction? Apparently, quite a lot, as both effects involve near-field phenomena. Even if two bodies are separated far enough so that particles cannot pass from one to the other, they're still linked through the fluctuating electromagnetic field and van der Waals interactions. Quantum fluctuations creating a temporary current density in one body induce

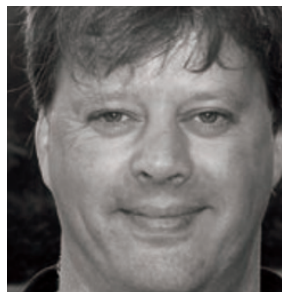

a correlated density in the other, creating a momentary attraction.

These fluctuating fields contribute to the anomalous heat transfer just described, and also create friction if the two bodies are in relative motion. As a recent review elegantly describes (A. I. Volokitin \& B. N. J. Persson,
Even if two bodies are separated, they're still linked...
Rev. Mod. Phys. 79, 1291-1329;

2007), the effect arises because motion induces a time lag between the current densities in the two bodies. This time lag creates, in addition to the attractive force between the bodies, a dissipative transverse force resisting their motion, a fundamental friction of quantum origin, existing even at absolute zero.

These effects, it seems, are more than merely hypothetical, and influence more than theoretically or experimentally 'ideal' surfaces. Recent experiments have detected frictional forces even for electrons moving within quantum wells, and we can expect more surprises as technology for tailoring atomic and nanoscale materials improves. The notion of friction may enter elementary mechanics in a disguise of utter simplicity, but we still have much to learn about one of the most pervasive phenomena of science.

Mark Buchanan

\section{A testable prediction}

We have used up nearly half of the oil nature made for us; we will start to run out relatively soon. We could then choose to ignore the consequences for our planet's climate and switch to heavy oil, shale oil, methane hydrate and coal to provide power for our world. But, given the prodigious rate at which we burn up the stuff, we will in any case start to run out of all fossil fuels by the end of this century.

Scientists are supposed to make predictions. Experiment or observation tests the prediction, and the fate of the scientist's theory - acceptance or rejection - rides on the outcome. That's how science works. I have a prediction to make. Here it is: civilization as we know it will come to an end some time in this century, when the fuel runs out.

This is different from normal scientific predictions in a crucial way. Usually, the scientist hopes that the prediction will prove to be correct, and merely making the prediction does not change the phenomenon in question. In this case, I do hope the prediction will be wrong, and that merely making the prediction will help

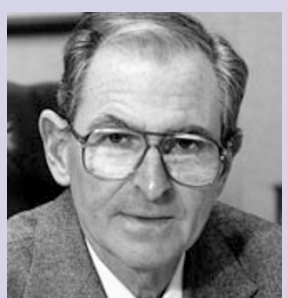

Civilization as we know it will come to an end some time in this century. to make it become wrong: perhaps you, reader, can join in tackling the big job before us, to kick the fossil-fuel habit before we run out.

Early lifeforms released oxygen into the atmosphere and buried carbon in the ground, preparing the planet for creatures like us. Now the planet is in our hands and, unlike early life, we are aware of our responsibilities and the possible consequences of our actions. What happens next is up to us.

David Goodstein 